\title{
Cooling techniques for excilamps driven by dielectric barrier discharge
}

\author{
Dmitry V. Schitz ${ }^{\mathrm{a}, *}$ Mikhail I. Lomaev, ${ }^{\mathrm{b}, \mathrm{c}}$ Viktor S. Skakun, ${ }^{\mathrm{b}}$ and \\ Viktor F. Tarasenko ${ }^{b}$ \\ ${ }^{a}$ Immanuel Kant Baltic Federal University, Science Park, Kaliningrad, Russia \\ ${ }^{\mathrm{b}}$ Siberian Branch of Russian Academy of Sciences, Institute of High Current Electronics, \\ Tomsk, Russia \\ ${ }^{\mathrm{c}}$ National State Research University, Faculty of Physics, Tomsk, Russia
}

\begin{abstract}
Our study describes effective techniques to transfer heat away from UV emitters based on dielectric barrier discharge excilamps. It presents findings from an investigation into the efficiency of excilamp radiation when cooled by air, inert gas, and liquid refrigerants. The devised cooling techniques were used to create radiation sources with a UV power density of up to $117 \mathrm{~mW} / \mathrm{cm}^{2}$. (C) The Authors. Published by SPIE under a Creative Commons Attribution 4.0 Unported License. Distribution or reproduction of this work in whole or in part requires full attribution of the original publication, including its DOI. [DOI: 10.1117/1.OE.60.5.057106]
\end{abstract}

Keywords: excilamp; UV and VUV light source; gas discharge; narrowband radiation; cooling techniques.

Paper 20210104 received Jan. 27, 2021; accepted for publication May 13, 2021; published online May 28, 2021.

\section{Introduction}

As interest in gas-discharge lasers wanes, the focus is gradually shifting to the development and study of efficient and intense sources of spontaneous narrow-band UV and VUV radiation, where the source of radiation is excimer and exciplex molecules excited in dielectric barrier discharge (DBD) plasma in inert gas or inert gas-halogen mixtures. Sources of this type are commonly referred to as barrier discharge excilamps, and they are widely used in various fields of science and technology. ${ }^{1}$

The efficiency of excimer or exciplex molecule radiation crucially depends on the design of a quartz bulb and electrodes of an emitter, the pressure and composition of operating gas, ${ }^{1}$ the excitation pulse shape, ${ }^{2}$ and the operating gas temperature. As temperature rises, the efficiency decreases monotonically because the dimer formation rate declines ${ }^{3}$ and dimer dissociation efficiency grows. ${ }^{4}$ Thus to obtain intense-radiation and high-efficiency excilamps, the operating gas should be rapidly cooled. Emitter overheating shortens the lifetime of electrodes in one-barrier lamps ${ }^{5}$ and causes a reduction in halogen concentration as a result of a heterogeneous chemical reaction between halogen atoms and the quartz wall in two-barrier excilamps. ${ }^{6}$ This paper aims to develop and test various ways of cooling actuating media of excilamps excited by electric barrier discharge.

\section{Air Cooling}

The simplest and most reliable technique of cooling an emitter is to decrease its temperature by air. In most cases, the outer surface of an emitter is air-cooled, and/or a strong air stream is passed through the inner tube if the emitter is co-axial. Figure 1 shows the directions of air streams coming from two centrifugal fans. One of them pumps air through the inner tube of the emitter, and the other delivers air to its center, where the air stream splits in two. From there, it runs throughout the length of the emitter and cools the outer surface. Tarasenko et al. described these excilamps in Ref. 7.

*Address all correspondence to Dmitry V. Schitz, DSchitz@kantiana.ru 


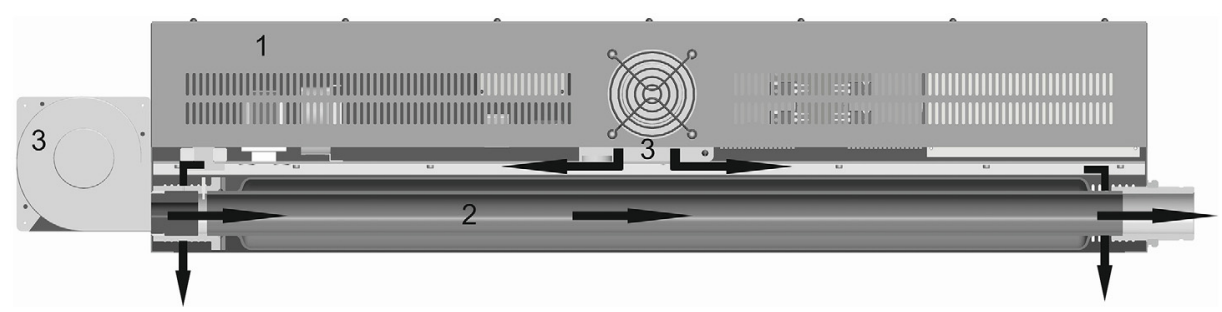

Fig. 1 Cooling an emitter of a portable excilamp by air: (1) excilamp housing with in-built power supply, (2) emitter, and (3) centrifugal fans. The arrows show the direction of air streams.

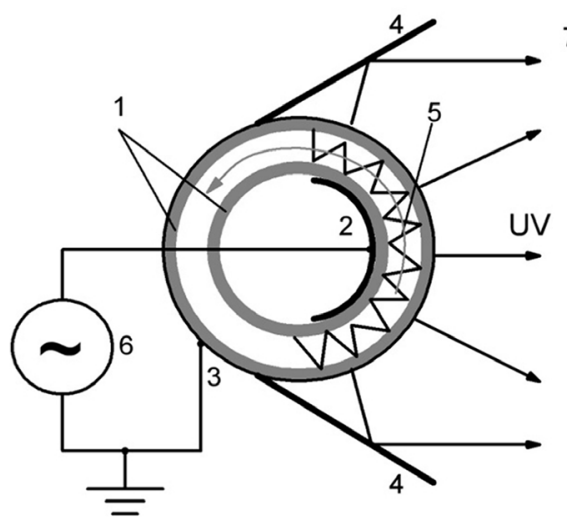

(a)

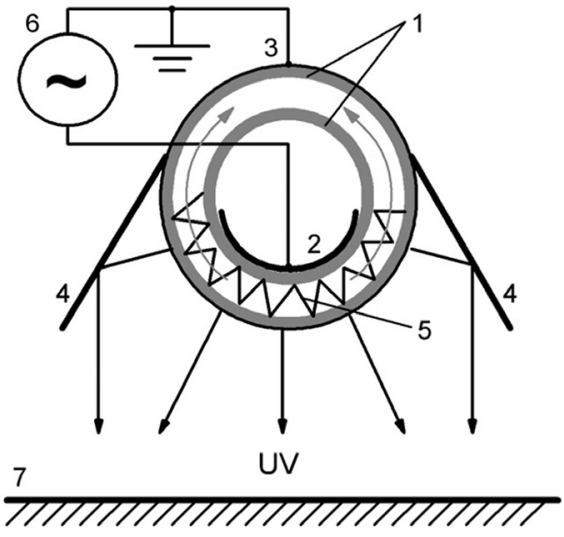

(b)

Fig. 2 Segmental discharge ignition in an emitter: (a) irradiation of vertical surface and (b) downward radiation. (1) Quartz tubes of emitter bulb, (2) potential electrode, (3) grounded electrode, (4) reflector, (5) discharge zone, (6) pulsed power supply, and (7) irradiated surface.

Irradiating surfaces often require using reflectors to form a unidirectional radiation flow. In such cases, it is advisable to ignite discharge on the side (in the segment) where the irradiated object is, rather than throughout the volume of the emitter (see Fig. 2).

An advantage of emitters with segmental discharge ignition is that they contain a buffer volume with operating gas, and the lifetime of a sealed-off emitter is increased this way. Segmental ignition also ensures the stirring of operating gas and prevents local overheating in the discharge area. The gas is stirred by convective fluxes; Fig. 2 shows their directions.

Heat transfer through an impenetrable wall is accounted for by the difference in temperatures between the hot and cold substances, the heat transfer coefficient, and the area of the wall surface. When the inner surface of the inner tube of an excilamp is air-cooled, the efficiency of heat transfer is determined by the initial air-stream temperature, the rate of the airflow, as well as the area of the cooled tube surface.

As mentioned above, in excimer lamps with two dielectric barriers, operating in a halogeninert gas mixture the major factor affecting the lifetime of a sealed-off emitter is the rate of the heterogeneous chemical reaction between halogen atoms and the quartz wall, which involves the formation of chlorosiloxanes $\mathrm{Si}_{n} \mathrm{O}_{n} \mathrm{Cl}_{2 n}$ ( $n=3$ to 5): ${ }^{6}$

$$
n \mathrm{SiO}_{2}(\text { solid })+2 n \mathrm{Cl}(\text { gas }) \rightarrow \mathrm{Si}_{n} \mathrm{O}_{n} \mathrm{Cl}_{2 n}(\text { solid })+(n / 2) \mathrm{O}_{2}(\text { gas }) .
$$

The rate constant of the heterogeneous reaction Eq. (1) $K$ depends on the temperature, as given by the Arrhenius equation: 


$$
K=A \cdot 10^{(-E a / k T)}
$$

In Eq. (2), $A$ is the pre-exponential factor, $E_{a}$ is the activation energy, $T$ is the absolute temperature, and $k$ is the Boltzmann constant. Equation (2) indicates that the rate constant and the rate of the heterogeneous reaction [Eq. (1)] depend exponentially on the temperature.

Spark and streamer gas discharge is associated with a greater chance of local heating of a fused quartz surface, as compared to diffuse discharge where the density of a current flowing through discharge plasma is evenly distributed across the wall of the emitter. The study of excilamp excitation regimes showed that higher radiation of exciplex molecules is caused by shortpulse excitation of operating gas as well as discharge formation via multiple micro-channels, each consisting of two diffuse cones connected at the apexes. ${ }^{2}$ This mechanism was studied by Tarasenko et al. ${ }^{8}$ This type of discharge is preferable to spark or streamer ones because current distribution is more even upon contact between diffuse micro-discharges and quartz walls. Local overheating of a fuse quartz wall is thus prevented.

\section{Liquid Cooling}

If the level of gas medium excitation is above $50 \mathrm{~mW} / \mathrm{cm}^{3}$, more effective heat transfer is ensured by cooling by liquid and/or air. ${ }^{9}$ Systems for liquid heat transfer from lamps can have a closed- or open-loop design. A closed loop is filled with distilled water or any other insulating refrigerant pumped through an emitter or a heat exchanger. The open-loop approach, within which tap water is used as the refrigerant, is the simplest. Yet the closed-loop design has several advantages: lower water consumption, no salt build-up on the walls of a cooled emitter, and the insulation of high-voltage electrodes.

When cooling co-axial emitters by a liquid, the refrigerant is usually pumped through the inner tube to cool the operating gas, whereas radiation is passed through a mesh electrode on the surface of the outer tube. To cool the outer tube, its surface is covered with radiators, whose temperature is decreased by water or air (Fig. 3). Thermal paste is applied to areas between the surface of the emitter and that of a metal radiator to increase the heat transfer coefficient.

Combined double-side cooling by air and water can be accomplished by forcing air through the inner cavity of the emitter and pumping water through the inner cavities of the emitterholding plate (Fig. 3).

In some cases, tap water cannot be used to cool DBD-driven excilamps since undistilled water is an electrolyte and effectively conduct a sine or square wave high voltage to a grounded electrode. At the same time, due to the low mobility of current carriers (ions and cations), water has high resistance when excilamp excided by alternating-polarity micro-second pulses.

This effect was not obtained when using sinusoidal voltage or unipolar-pulsed voltage.
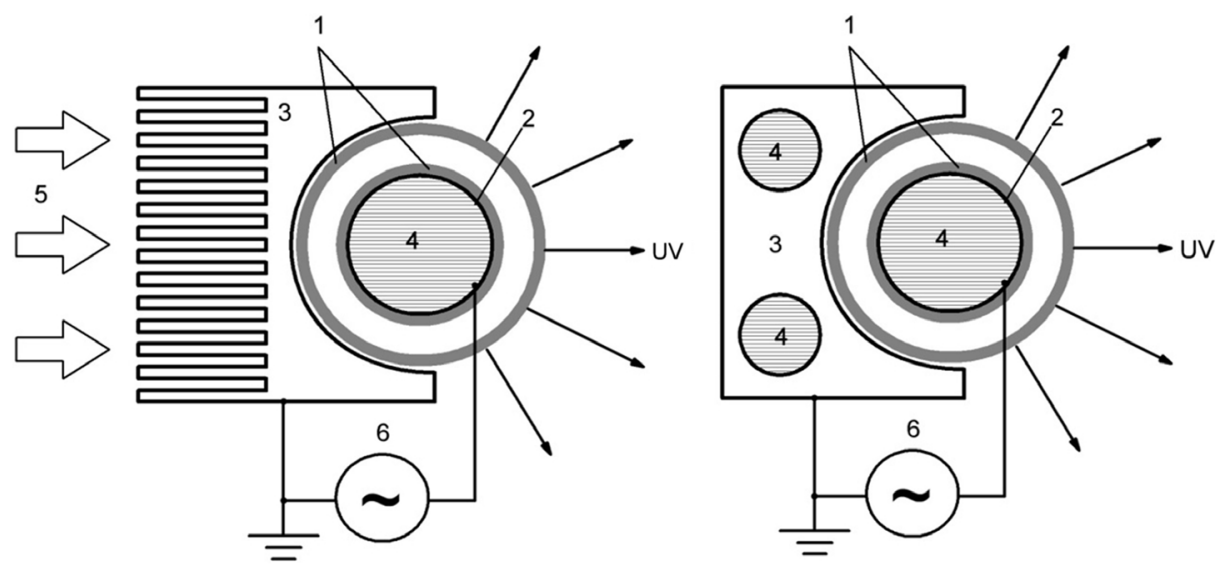

Fig. 3 Double-side cooling of a co-axial emitter: (1) quartz tubes, (2) potential electrode, (3) metal radiator, (4) water flow, (5) air stream, and (6) pulsed power supply. 


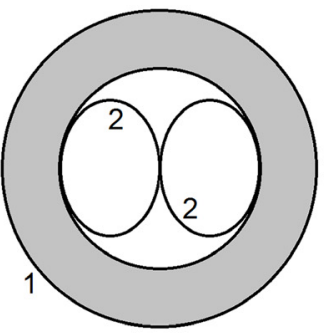

(a)

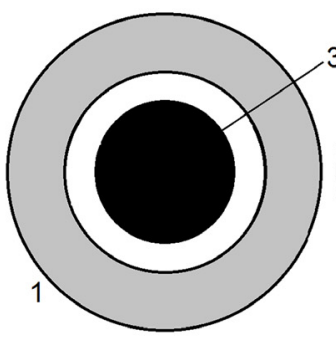

(b)

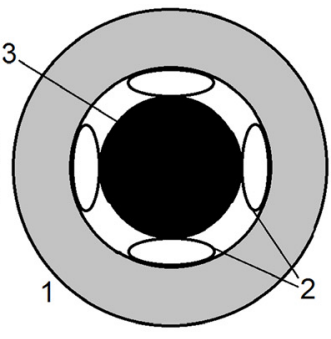

(c)

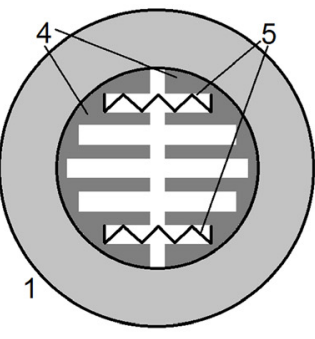

(d)

Fig. 4 Ways to cool the inner tube of a quartz bulb of a co-axial structure: (1) quartz bulb, (2) aluminum foil tubes, (3) metal tube, (4) ribbed radiator, and (5) metal spring. (a) two aluminum foil tubes, (b) solid metal tube, (c) solid metal tube with four aluminum foil tubes, and (d) ribbed metal radiator.

\section{Optimization of a Cooling System}

In high-power excilamps, which cannot be cooled by liquid, it is recommended to use strong air streams directed at the outer-tube radiator as well as to pump air rapidly through the inner tube of an excilamp.

Six types of radiators placed into the inner tube were used in the experiment for optimising the structure of excilamp cooling systems (Fig. 4). These were: (a) two 50- $\mu$ m-thick aluminum foil tubes; (b) metal tubes of a diameter of 10, 15, and $18 \mathrm{~mm}$; (c) a metal tube of a diameter of $15 \mathrm{~mm}$ enclosed in several aluminum foil tubes; and (d) an aluminum radiator. A xenon-dimer excimer lamp with a wavelength of $172 \mathrm{~nm}$ had a quartz bulb of $\varnothing 40 \times 250 \mathrm{~mm}$ (the working length of the surface was $200 \mathrm{~mm}$ ). The power supplied to the emitter was constant at $280 \mathrm{~W}$. The output power density was measured using a Hamamatsu C9536 meter with an H9535-172 sensor placed $5 \mathrm{~mm}$ away from the surface of the emitter to prevent UV sensor overheating. The gap between the sensor and the surface was purged with a flow of nitrogen. The temperature of the outer surface of the emitter was measured by a thermocouple.

Air was pumped through a quartz bulb of a diameter of $23 \mathrm{~mm}$ at rates of 10,20 , and $40 \mathrm{l} / \mathrm{min}$. Figure 5 illustrates the dependence of the temperature of the surface of an excimer lamp and power density on the airflow rate and operating time for the lamp with the inner electrode made of a metal tube of a diameter of $15 \mathrm{~mm}$. Low-rate air streams cause a quartz bulb to heat to higher temperatures. This has a significant effect on the radiated power: the higher the rate of the airflow is, the greater is the power radiated by an excilamp, and therefore, its efficiency.

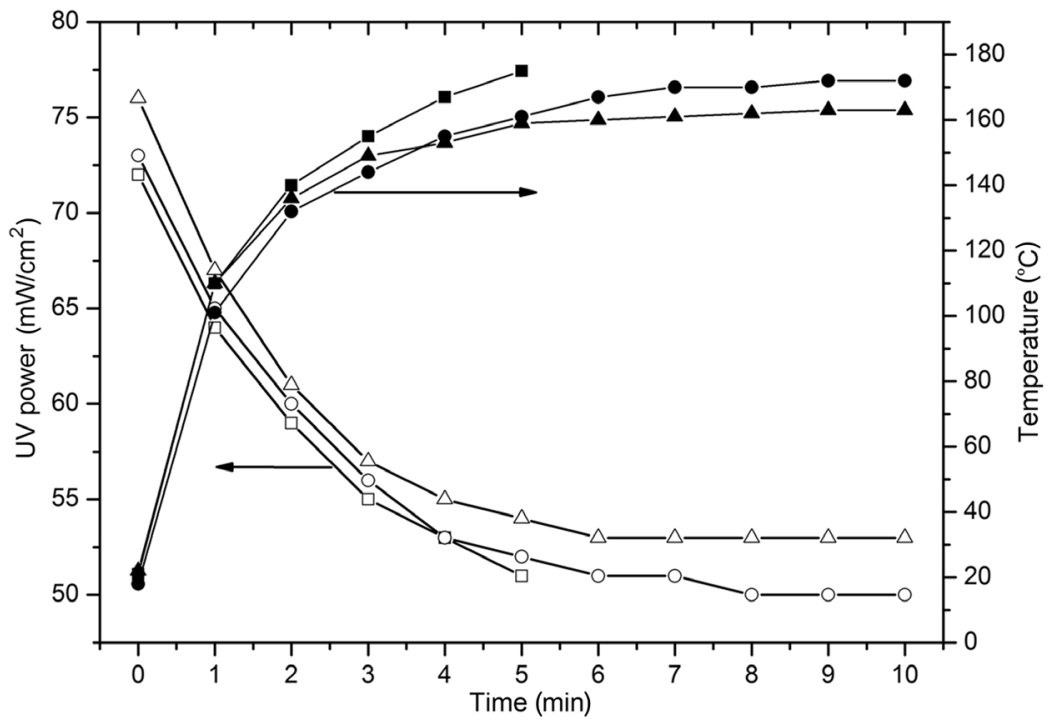

Fig. 5 The dependence of the temperature of an excimer lamp and UV power density (172 $\mathrm{nm}$ ) on time and the airflow rate through the inner tube: $\boldsymbol{\|}$ and $\square, 10 \mathrm{l} / \mathrm{min} ; \bullet$ and $\bigcirc, 20 \mathrm{l} / \mathrm{min}$; and $\boldsymbol{\Delta}$ and $\triangle, 40 \mathrm{l} / \mathrm{min}$. 


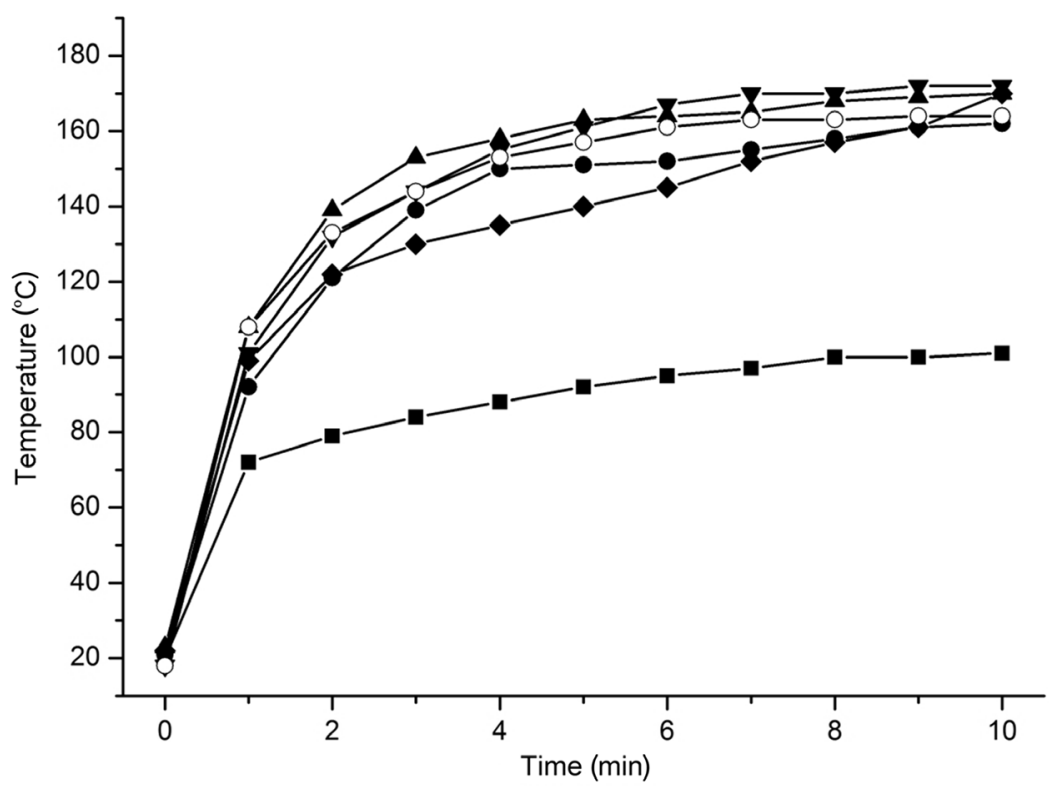

Fig. 6 The dependence of the temperature of the outer wall of a $\mathrm{Xe}_{2} *$ excilamp on operating time and the radiator type at air flow rate $20 \mathrm{l} / \min : \bullet$, radiator $a$; $\mathbf{\Lambda}$, radiator $b$ with a $10-\mathrm{mm}$ tube; $\mathbf{\nabla}$, radiator $b$ with a 15-mm tube; $\diamond$, radiator $b$ with an 18-mm tube; $\bigcirc$, radiator $c$; and $\mathbf{m}$, ribbed metal radiator $d$.

Figure 6 demonstrates the dependence between the temperature of the outer wall of a quartz bulb and the type of radiator in the inner tube of an emitter. It can be seen that $a, b$, and $c$-type radiators have a relatively low heat removal rate. The temperature of the excilamp reached $150^{\circ} \mathrm{C}$ to $170^{\circ} \mathrm{C}$ as a result.

The most effective cooling in the inner quartz tube of an excimer lamp was observed when using a ribbed aluminum radiator $d$. The large area of contact between the quartz glass and the $d$-type radiator, the high thermal conductivity of aluminum, and the wide area of the surface radiator ribs ensure a maximum decrease in the temperature of the excilamp and a relatively high radiated power (Fig. 7).

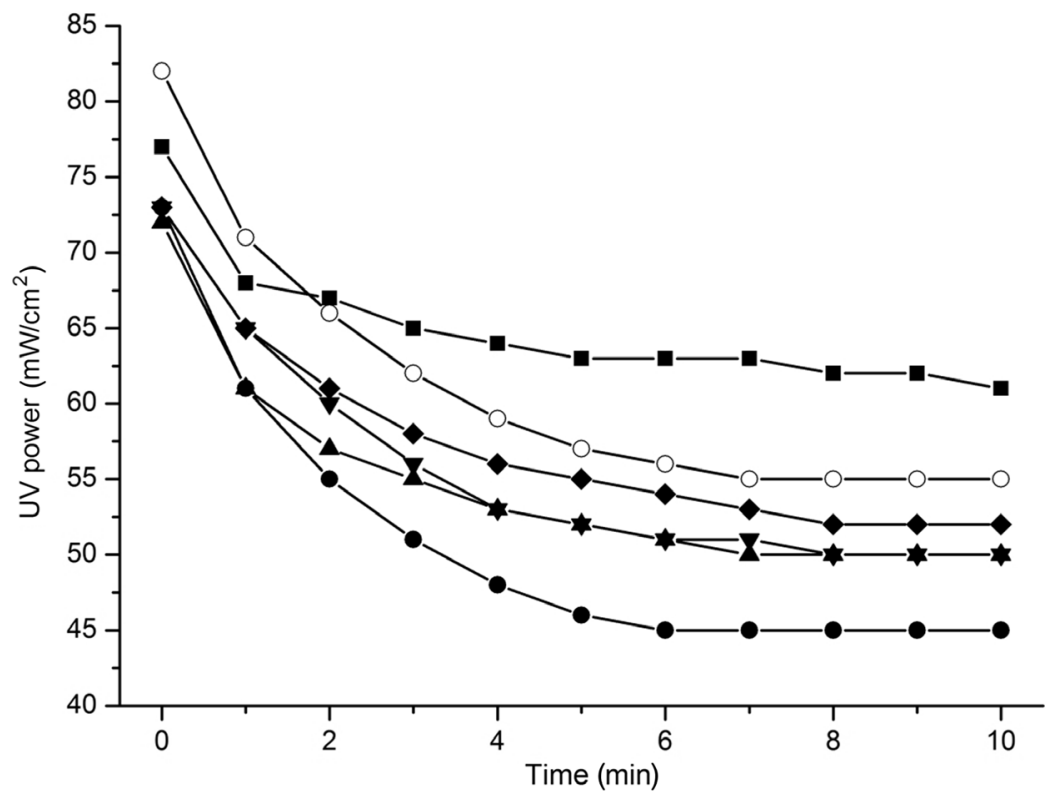

Fig. 7 The dependence between radiated power $(172 \mathrm{~nm})$ and the type of radiator at air flow rate $20 \mathrm{I} / \mathrm{min}: \bullet$, radiator $a$; $\mathbf{\Lambda}$, radiator $b$ with a $10-\mathrm{mm}$ tube; $\boldsymbol{\nabla}$, radiator $b$ with a $15-\mathrm{mm}$ tube; $\bullet$, radiator $b$ with an 18-mm tube; $O$, radiator $c$; and $\mathbf{m}$, ribbed metal radiator $d$. 


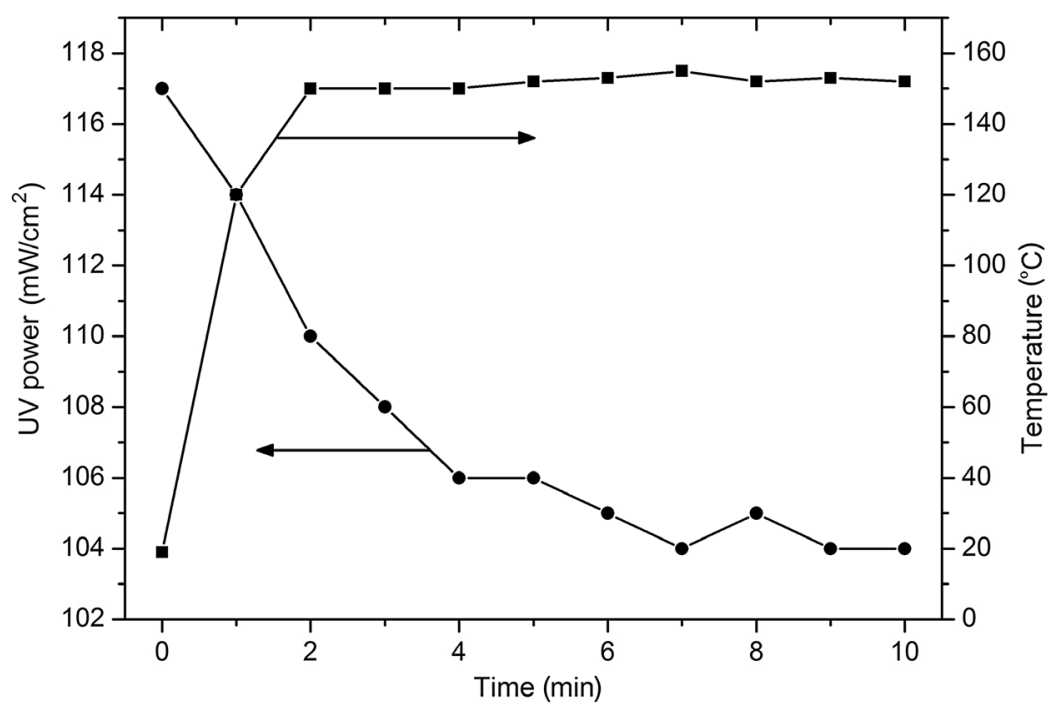

Fig. 8 The dependence of the excimer lamp temperature and UV power density $(172 \mathrm{~nm})$ on time for a ribbed metal radiator $d$ at airflow rate through the inner tube $40 \mathrm{l} / \mathrm{min}$.

A ribbed radiator caused the temperature of the outer surface of an excimer lamp to stay at or below $100^{\circ} \mathrm{C}$. This efficient cooling technique made it possible to increase the excitation power of an excimer lamp to $350 \mathrm{~W}$. In this case, after $10 \mathrm{~min}$ of the excilamp operation, the temperature of the outer wall of the emitter stabilized at below $160^{\circ} \mathrm{C}$. As previously stated, to avoid the overheating of the UV meter during the experiments, a UV sensor was positioned $5 \mathrm{~mm}$ away from the radiating surface of the excilamp. To obtain the absolute values of the UV power density, the sensor was briefly placed on the surface of the excilamp. Fig. 8 shows changes in the temperature of the outer surface of the excilamp and the intensity of its radiation within $10 \mathrm{~min}$. The maximum power of $117 \mathrm{~mW} / \mathrm{cm}^{2}$ was observed upon switching on the excilamp. After that, it decreased and flatlined at $104 \mathrm{~mW} / \mathrm{cm}^{2}$.

\section{Excimer Lamp with a High VUV Power Density}

These findings aided in creating a xenon-dimer-based modular excimer lamp with a high VUV power density $117 \mathrm{~mW} / \mathrm{cm}^{2}$ (Fig. 9). The excimer lamp consisted of six co-axial emitters

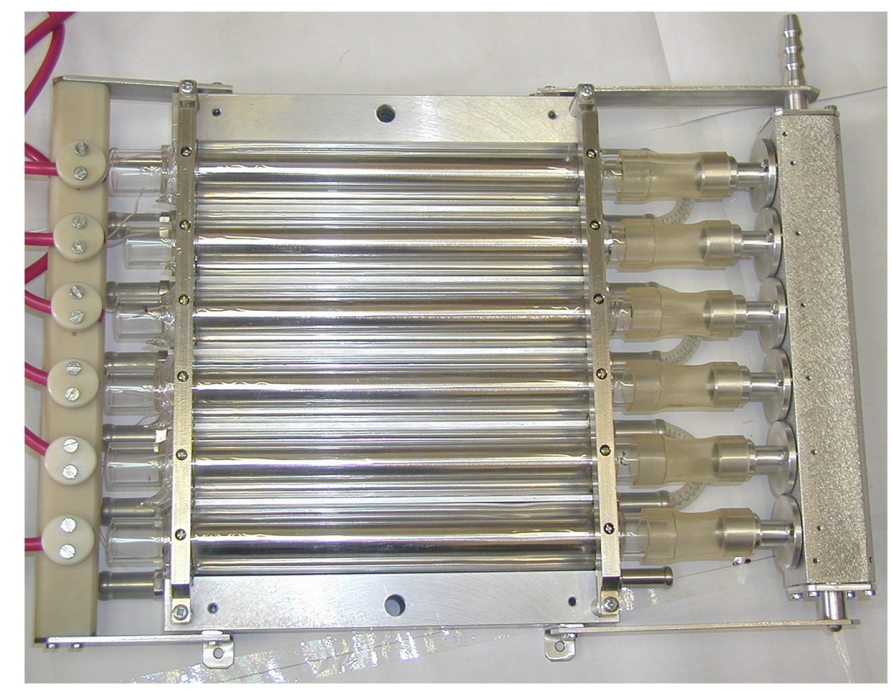

Fig. $9 \mathrm{~A} \mathrm{Xe}_{2} *$ dimer excimer lamp with six coaxial emitters. 
excited by DBD and mounted on a water-cooled metal radiator. The inner tubes of all six emitters were cooled by air. The area of the radiating surface was $20 \times 20 \mathrm{~cm}^{2}$. The total excitation power for six emitters was $2.1 \mathrm{~kW}$.

\section{Conclusion}

This work described several ways to cool excilamps by liquid (water) or air flow and by a mixed technique. The highest efficiency of a DBD-driven xenon excilamp was obtained when using an aluminum radiator with water flow inside it to cool the outer wall of the excilamp, as well as when employing an inner ribbed metal radiator cooled by an intensive airflow. Effective cooling enables the work of excilamps at a high excitation power per unit of volume without overheating. When exciplex lamps are used, effective cooling extends the life of the halogen-inert gas mixture.

\section{Acknowledgments}

This work was partially supported by the Ministry of Science and Higher Education of the Russian Federation (Grant No. FZWM-2020-0003).

\section{References}

1. M. I. Lomaev et al., "Excilamps: efficient sources of spontaneous UV and VUV radiation," Phys.-Uspekhi 46(2), 193-209 (2003).

2. M. I. Lomaev et al., "Influence of excitation pulse form on barrier discharge excilamp efficiency," Proc. SPIE 4460, 38-45 (2002).

3. F. Marchal et al., "Formation of xenon excimer between 200 and $300 \mathrm{~K}$ following selective excitation of the Xe (6s) metastable state," in 28th Int. Conf. Phenomena Ionised Gases, J. Schmidt, Ed., Prague, Czech Republic, pp. 100-103 (2007).

4. M. Salvermoser and D. E. Murnick, "Efficient, stable, corona discharge $172 \mathrm{~nm}$ xenon excimer light source," J. Appl. Phys. 94(6), 3722-3731 (2003).

5. S. M. Avdeev et al., "Two-band emission source based on a three-barrier $\mathrm{KrCl}-\mathrm{XeBr}$ excilamp," Tech. Phys. Lett. 34, 725-727 (2008).

6. M. V. Erofeev et al., "Lifetime of working mixtures of $\mathrm{XeCl}$ and $\mathrm{KrCl}$ excilamps," Atmos. Oceanic Opt. 13(3), 286-288 (2000).

7. V. Tarasenko et al., "UV and VUV excilamps with high peak power," J. Light Visual Environ. 35(3), 227-233 (2011).

8. V. F. Tarasenko, D. V. Schitz, and M. I. Lomaev, "About the formation of a barrier discharge in a $\mathrm{KrCl}$ excilamp," Russ. Phys. J. 46(7), 745-747 (2003).

9. E. Arnold, R. Dreiskemper, and S. Reber, "High-power excimer sources," in 8th Int. Symp. Sci. and Tech. Light Sources, G. Babucke, Ed., Greifswald, Germany, pp. $90-98$ (1998).

Dmitry V. Schitz received his doctoral degree from Tomsk State University in 2003. He is a head of the Optics Laboratory of the Science Park at the Immanuel Kant Baltic Federal University. He is the author and coauthor of more than 60 research publications and 20 patents on high-power and high-efficiency dielectric barrier discharge excilamps for medical and industrial applications. He has considerable experience in the development of power supplies for gasdischarge light sources.

Mikhail I. Lomaev is a senior researcher of the Institute of High Current Electronics at Siberian Branch of the Russian Academy of Sciences and a professor of the Faculty of Physics at the National Research Tomsk State University. His research interests are focused on high-power excilamps and diffuse pulsed discharge formed by run-away electrons. He has more than 300 publications, including 22 patents and 4 monographs.

Viktor S. Skakun received his doctoral degree from Tomsk State University in 1993. He is a senior researcher of the Institute of High Current Electronics at the Siberian Branch of the 
Russian Academy of Sciences. His fields of specialization are UV and VUV gas-discharge light sources, high-current electronics, and electron beams generation. He authored more than 200 scientific articles and has 34 patents.

Viktor F. Tarasenko is the founder and professor emeritus of the Laboratory of Optical Radiation at the Institute of High Current Electronics. He is the author and coauthor of more than 1000 journal publications and numerous conference papers. Under his leadership, a whole series of UV and VUV excilamps with record radiation characteristics was created and the foundations of their application were laid. Based on inductive energy storage generators, he created a number of pulsed gas lasers having the highest physical efficiency and pulse durations. He is the initiator of many unique developments including the study of runaway electrons. 